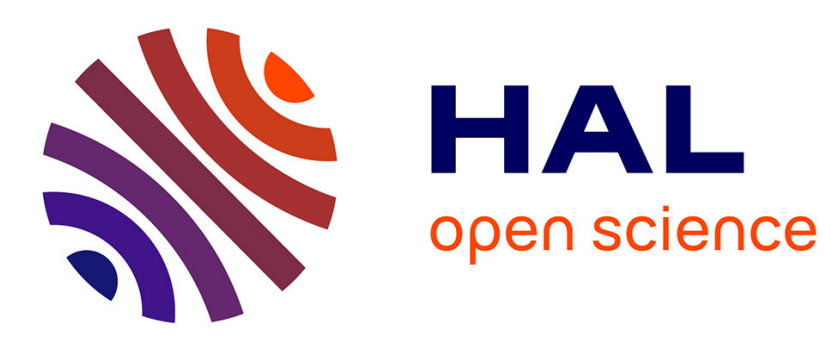

\title{
Integrated Routing and Storage for Messaging Applications in Mobile Ad Hoc Networks
}

\author{
Delphine Nain, Noshirwan Petigara, Hari Balakrishnan
}

\section{To cite this version:}

Delphine Nain, Noshirwan Petigara, Hari Balakrishnan. Integrated Routing and Storage for Messaging Applications in Mobile Ad Hoc Networks. WiOpt'03: Modeling and Optimization in Mobile, Ad Hoc and Wireless Networks, Mar 2003, Sophia Antipolis, France. 10 p. inria-00466607

\section{HAL Id: inria-00466607 https://hal.inria.fr/inria-00466607}

Submitted on 24 Mar 2010

HAL is a multi-disciplinary open access archive for the deposit and dissemination of scientific research documents, whether they are published or not. The documents may come from teaching and research institutions in France or abroad, or from public or private research centers.
L'archive ouverte pluridisciplinaire HAL, est destinée au dépôt et à la diffusion de documents scientifiques de niveau recherche, publiés ou non, émanant des établissements d'enseignement et de recherche français ou étrangers, des laboratoires publics ou privés. 


\title{
Integrated Routing and Storage for Messaging Applications in Mobile Ad Hoc Networks
}

\author{
Delphine Nain, Noshirwan Petigara, and Hari Balakrishnan \\ Massachusetts Institute of Technology \\ Cambridge, MA 02139 \\ \{delfin,nosh\}@alum.mit.edu,hari@mit.edu
}

\begin{abstract}
This paper is motivated by the observation that traditional ad hoc routing protocols are not an adequate solution for messaging applications (e.g., e-mail) in mobile ad hoc networks. Routing in ad hoc mobile networks is challenging mainly because of node mobility - the more rapid the rate of movement, the greater the fraction of bad routes and undelivered messages. For applications that can tolerate delays beyond conventional forwarding delays, we advocate a relay-based approach to be used in conjunction with traditional ad hoc routing protocols. This approach takes advantage of node mobility to disseminate messages to mobile nodes. The result is the Mobile Relay Protocol (MRP), which integrates message routing and storage in the network; the basic idea is that if a route to a destination is unavailable, a node performs a controlled local broadcast (a relay) to its immediate neighbors. In a network with sufficient mobility_precisely the situation when conventional routes are likely to be non-existent or broken-it is quite likely that one of the relay nodes to which the packet has been relayed will encounter a node that has a valid, short (conventional) route to the eventual destination, thereby increasing the likelihood that the message will be successfully delivered. Our simulation results under a variety of node movement models demonstrate that this idea can work well for applications that prefer reliability over latency.
\end{abstract}

\section{Introduction}

Mobile ad hoc networks are useful in a number of situations where a deployed infrastructure using cellular base stations is inconvenient, expensive, or impossible. Examples of this include disaster relief or emergency response, autonomous teams of robots in a remote area, sensor networks, and military networks. In these situations, nodes in an ad hoc network move from time to time, often across a large area, while also cooperatively routing packets for other nodes.

Routing in ad hoc networks is challenging mainly because of node mobility. If nodes were mostly static, then traditional routing approaches from wired networks could be brought to bear on the problem, with occasional mobility being handled as an "uncommon" case in the same way that link failures in traditional wired networks are handled. Current approaches to mobile routing, broadly classifiable into proactive and re- active (on-demand) protocols, work toward providing a connected routing topology in the face of node mobility. Proactive protocols maintain routes via a background process (e.g., using distance-vector updates) [8], while reactive protocols discover routes on-demand when they need to communicate with another node more than one hop away $[8,7]$. The performance of both types of protocols, measured in terms of the number of packets successfully delivered to the total number of packets sent (the packet delivery ratio), degrades as the rate of node movement increases [1].

We make the observation that not all applications in mobile ad hoc networks require the same "best-effort" semantics offered by an IP-based ad hoc network, where a packet not delivered within a small amount of time (on the order of a small number of round-trip times) is simply discarded. Messaging applications, such as e-mail, are an important class of applications in these networks; for these applications, the eventual delivery of a message is more important than any other metric of performance such as delivery latency. This paper addresses the problem of designing a message routing protocol to support such applications in an ad hoc network.

Our approach to this problem takes advantage of node mobility, rather than treat movement as a problem that needs to be tackled and overcome. To improve the delivery ratio of packets in the network, we use relay nodes; under various circumstances, when a node decides that it does not have a good route to the destination and is unlikely to obtain one, it distributes the message to one or more of its immediate neighbors, which store the message for a while. In a network with sufficient mobility-precisely the situation when conventional routes are likely to be non-existent or broken-the hope is that one of the relay nodes to which the packet has been distributed will encounter a node that has a valid route to the eventual destination, thereby increasing the likelihood that the message will be successfully delivered to the destination.

Of course, this increased reliability in terms of the packet delivery ratio comes at the expense of increased latency of message delivery, but this is a good trade-off for messaging applications. The challenge, however, is to ensure that such relaying can be done without using up a large amount of network bandwidth or node storage. We give the general design principles of the Message Relay Protocol, MRP, as well as our 
particular implementation of the MRP protocol when used in conjunction with the DSDV protocol (the MRP-enhanced DSDV protocol). We show using simulations that an MRPenhanced DSDV protocol increases the average delivery ratio by $31 \%$ compared to DSDV, while using less than $1 \%$ of extra bandwidth and a relatively small amount of extra node storage space (40 packets). We evaluate MRP under a variety of movement models, node densities and message transmission rates and show that the MRP protocol scales well, demonstrating that its overall performance is not affected by node density, node movement, or message transmission rates. Our results indicate that MRP can be a viable substrate on which messaging and email-like applications in ad hoc networks can be layered. In addition, an attractive property of MRP is that it achieves these goals without assuming any knowledge of node position or any knowledge of node movement vectors.

\section{Related Work}

Simulations of proactive (e.g., DSDV [8]), reactive (e.g., DSR [3], TORA [7]), and hybrid (e.g., AODV [9]) ad hoc routing protocols demonstrate that the packet delivery ratio degrades when mobility is high because of route instability and frequent network partitions [1]. However, the methods proposed to overcome these problems fall under the category of timeout-based retransmissions solutions, which we argue are not the best substrate over which to build messaging applications in ad hoc networks. For example, DSR has a mechanism called "route maintenance," by which the sender is notified that a route is no longer valid. Upon receiving such a message, it can then retransmit the packet with a cached or newly discovered route. Similarly, when DSDV detects a broken route, it stores the packet until the next route update at which time it tries to resend the packet. In contrast to these approaches, we find that the packet delivery ratio can be greatly improved by using the idea of relaying.

Rus and $\mathrm{Li}$ present routing algorithms using the relay nodes [5]. However, their assumptions are significantly different than ours. First, they assume that there is knowledge of either movement trajectories and/or node positions. Our algorithm does not assume knowledge of these. Second, their definition of a relay node is based on the the concept of mobile agents[4]. These nodes can move under their own control depending on where the message has to be delivered. In contrast, our mobile nodes cannot be controlled to move along particular paths; rather, they move randomly through the network independent of the requirements of the relaying protocol. Rus and $\mathrm{Li}$ work within their more controlled framework to guarantee delivery of packets by modifying the trajectories of relay nodes. We relax these constraints to show how the concept of relay nodes can increase delivery ratio even without actively constraining node movement along particular trajectories. We envision our algorithm to be useful in environments where there is no knowledge of positions and trajectories, and where one cannot easily predict or control where nodes move.

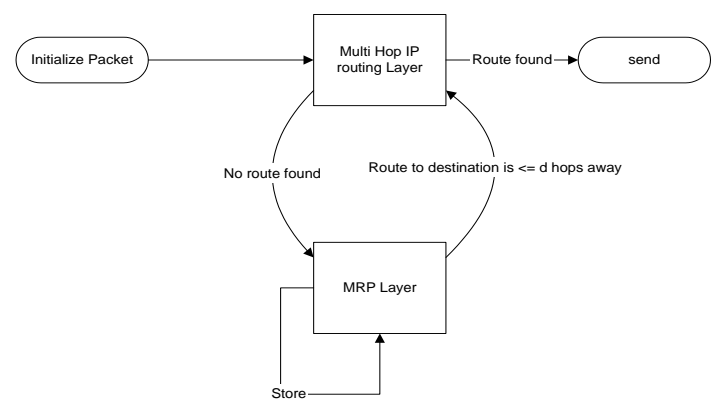

Figure 1: Integration of the MRP Protocol with a traditional ad hoc IP Routing Protocol

Grossglauser and Tse have explored a theoretical framework [2] where $n$ nodes with infinite buffer move independently around the network and every node gets close to any other node for $1 / n$ seconds per time slot. Within this framework, a node $s$ gives a message addressed to node $t$ to another randomly chosen node one hop away in the network called a "receiver." When the receiver happens to be one hop away from the destination node $t$, it gives it the message. Hence a message will only make two hops and no message will be transmitted more than twice. This ensures that the bandwidth used is minimal and that the long-term throughput per senderreceiver pair can be kept constant $(O(1))$ even as the number of nodes per unit area increases. Their other result is that a message is guaranteed to be delivered, even if its delivery time is averaged over many time slots. This result sets a theoretical bound, since it assumes a complete mixing of the trajectories so that every node can get close to another one. This motivates our work since we believe that there is an opportunity to trade-off delay for message delivery ratio in mobile networks by using intermediary relay nodes. We also add temporary storage to the primitive operations allowed, and produce a practical protocol based on the idea of integrating storage with routing.

\section{The Mobile Relay Protocol}

The Mobile Relay protocol (MRP) is designed to be layered on top of an existing ad hoc routing protocol and is responsible for the forwarding, storage and delivery of relay packets. We first describe the general MRP protocol and how it integrates with different types of ad hoc routing protocols. Then, we describe in more detail our particular implementation of MRP when used in conjunction with DSDV [8].

\subsection{Overview}

Figure 1 shows the forwarding of a packet by an MRPenhanced ad hoc routing protocol. If the traditional routing step fails to find a route for the packet, the packet is handed over to the MRP layer. The MRP layer broadcasts that packet locally to the node's immediate neighbors, who become relay nodes for that packet. Each relay node may store that packet until it discovers an IP route to the packet's destination according to the IP routing protocol being used. When a relay 


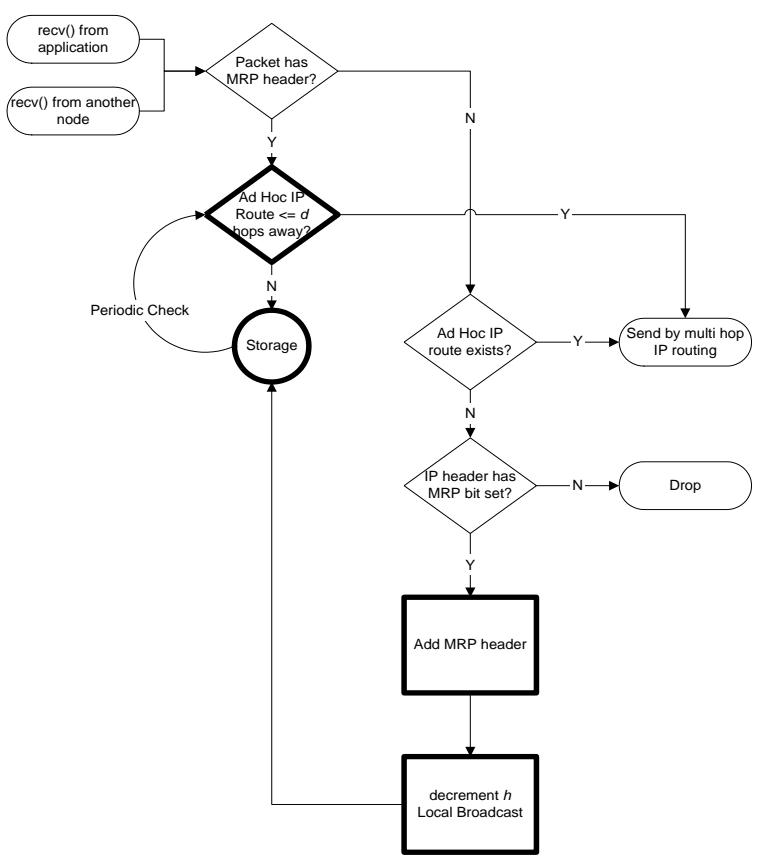

Figure 2: MRP Protocol: Forwarding

node finds a route less than $d$ IP hops away for a relay packet it has in storage, it passes it back to the traditional routing layer that forward it to its destination. $d$ is a protocol parameter $\geq 1$; we find in our simulations that $d=1$ provides good performance, implying that the transition from MRP back to using an IP route can be done "late," when one of the relay nodes comes within radio range of the intended destination.

Using MRP is optional. Whether MRP is used depends on information in the transport protocol and IP headers, which allows applications or the network operator to choose if packets can afford to be relayed if reliability matters (e.g., a messaging application packet), or whether they should not be relayed because delivery order and latency is more important than reliability (e.g., for interactive traffic, audio, etc.).

Figures 2 and 3 summarize the interaction between the MRP layer (bold boxes) and the traditional ad hoc IP routing layer (regular boxes). The rest of this section discuss how a packet transitions from traditional IP forwarding to MRP relaying, how packet relaying works, and how the protocol reverts to IP forwarding for final delivery.

\subsection{IP forwarding $\rightarrow$ MRP Relaying}

With traditional IP forwarding, the network layer at a node can receive three types of packets:

1. A packet sent by an application/transport layer running on the node that needs to be forwarded to its destination.

2. A packet sent by another node that doesn't match the IP address of the receiving node, which needs to be forwarded to its destination.

3. Other protocol-specific packets, such as route updates or route discovery packets. These packets are not relayed by MRP, since they typically require low-latency delivery.

When the routing layer receives a packet of the first two types, it checks if it has an MRP header. If it does not already have an MRP header, then the node needs to check if a route exists, or can be found, or if the packet needs to be relayed via MRP. If the packet does not already have an MRP header, the IP ad hoc routing layer uses a protocol-specific mechanism to discover a route for the packet. For example, this might be a standard forwarding table lookup (in proactive protocols) or a route discovery mechanism (in reactive protocols). If this mechanism succeeds, the packet is forwarded on as usual. If it fails, then the packet is handed over to the MRP layer.

At this stage, the packet has no MRP header. When the MRP layer receives such a packet, it first checks whether the packet may be relayed using MRP. There are several ways of doing this: one way might use a layer-4 classification with rules that decide whether MRP is suitable or not; another way might be to have the originating application or node set a bit in the IP header for the packet. If the packet is deemed unsuitable for MRP, then the packet simply loops back to the IP forwarding layer, which handles it like a regular packet for which no route is known (most protocols drop the packet and some store it for later attempts).

The interesting case for MRP is if the packet is deemed suitable for relaying using MRP. If so, an MRP header is added to the packet. The MRP header incorporates the IP address of the original sender and the final destination from the IP layer, and adds two other fields, $h$ and $d$.

1. $h$ specifies a running count of the number of remaining allowed relay hops, and decrements every time another relay operation is done. If $h$ becomes 0 , no further relays are allowed. $h$ is initialized to a value that depends on the expected movement range of nodes in the network. Section 3.3 describes the details of how this is done.

2. $d$ specifies how close to the eventual destination, in IP hops, might a relay node arrive before reverting from MRP to IP forwarding to have the packet delivered. For proactive routing protocols, $d \geq 1$ makes sense, since a background process will cause routes to destinations to be updated as nodes move. For reactive protocols, all we can reliably count on is $d=1$-when a relay node is within one hop of the destination, it will learn of this and can revert to IP forwarding to deliver the packet.

After the addition of the MRP header, the packet is locally broadcast by the node to its immediate neighbors. All nodes that receive this packet store it and go to the relaying step, described next. We emphasize that this is the only time in MRP that a local broadcast is done for any packet-a packet that already has an MRP header is never rebroadcast during subsequent relaying steps. 


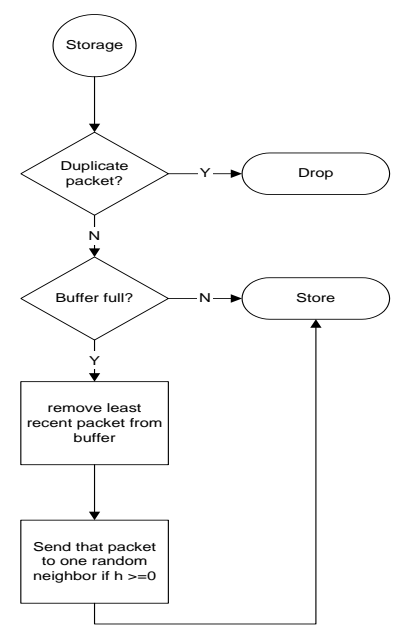

Figure 3: MRP Protocol: Storage

\subsection{Relaying}

If a packet received at the routing layer already has an MRP header, the packet is handed over to the MRP layer directly (Figure 2). This situation happens either when a node receives an MRP packet to relay from another node or an MRP packet to forward from the MRP layer directly.

The MRP layer first checks with the routing layer to see if there exists a route of less than $d$ hops to forward the packet. If so, it forwards the packet and the relay MRP packet is delivered. If no valid route exists for the packet, it enters the storage phase (Figure 3), which consists of the following steps:

1. If the packet is already stored in the node's buffer, then the older version of the packet is discarded.

2. Otherwise, the storage buffer is checked. If it is not full, then the packet is stored and the $h$ parameter in the MRP header of the packet is decremented by 1 .

3. If the buffer is full, then the least recent packet is removed from the buffer and it is relayed to a single random neighbor as long as the $h$ parameter in the MRP header of the packet is greater than 0 .

In this design, a packet is eliminated from a node only when the node runs out of buffer space for packets it is currently relaying. This means that the only time a packet is eliminated from the system is if all the nodes currently relaying it run out of space. It is conceivable that a mechanism where the original sender specifies a time-to-live field in seconds, with relaying nodes eliminating the packet when this time elapses, might prove useful. Our mechanism is simpler and demanddriven, and we think that the semantics of most messaging applications are probably not worth the additional complexity of maintaining real time.

\section{4 $\quad$ MRP Relaying $\rightarrow$ IP Forwarding}

When a packet is stored, the MRP layer needs a mechanism to routinely check if a route has been found for any packets in the relay buffer. The details of this mechanism are specific to the routing protocol being used.

\subsection{MRP-enhanced DSDV}

In this section, we describe how MRP can enhance DSDV. Our goal is not to create a better DSDV protocol, but rather to show that MRP can help improve DSDV's packet delivery ratio for latency-tolerant messaging applications during times of high mobility by taking advantage of node movement.

In DSDV, each node maintains a routing table that contains the next hop for each known (reachable) node on the network. Nodes synchronize their routing table by broadcasting periodic routing table update messages.

\subsubsection{MRP Relaying with DSDV}

The address resolution protocol (ARP) resolves an IP address to a hardware (MAC) counterpart and caches these bindings. For uncached bindings, most ARP implementations store the packet for which they are currently seeking ARP resolution. In ns-2 (our simulation platform), and indeed in many real systems, the ARP buffer only stores one packet, so consecutive packets for the same destination will cause all packets but one to be dropped until the ARP resolves the MAC address. To solve this problem, we paced the rate at which a relay node sends its relay packets so that there is no collision in the ARP cache. This proved to be insufficient in some cases, so we added an ARP callback where the link layer could tell the MRP layer which packets were dropped. The MRP layer then handled the packet as described before.

\subsection{2 $M R P \rightarrow I P$ Forwarding in MRP/DSDV}

Since a mechanism for frequent route updates is part of the DSDV protocol, it is simple for the MRP layer to check if it has a relay message stored for any node whose route has been updated and is less than or equal to $d$ hops.

When the DSDV layer discovers a valid route to a new destination through a route update, it notifies the MRP layer. If the MRP layer has any relay packets stored for that destination, and the destination is $d$ hop away, the MRP layer sends the packet back to the DSDV layer that is then responsible for forwarding it.

\section{Evaluation}

The overall goal of our experiments is to measure the ability of MRP-enhanced DSDV to deliver packets under a variety of movement models and compare its performance to DSDV.

We evaluate protocol performance using two metrics:

- Packet delivery ratio: The ratio between the number of packets originated by the application layer of the CBR (constant bit rate) sources and the number of packets received by the CBR sink at the final destination. 
- Packet latency: The time taken from when the packet leaves the application layer CBR source until it reaches the CBR sink at the final destination ${ }^{1}$.

We evaluate scalability using three parameters:

- Node Density: We vary the number of nodes for the given simulation area.

- Routing Overhead: We measure the overhead created by the MRP protocol and compare it to the overhead of DSDV. The overhead is calculated by measuring the total number of local broadcasts and the number of duplicate relay packets received at the destination.

- Message Rate: We vary the message rate to see how MRP scales to accomodate increasing message loads.

\subsection{Simulation Environment}

Our simulations were run on ns version $2.14 \mathrm{~b}$ [6], which provides support for simulating both the physical aspects and protocol layers for multi-hop wireless networks. It provides implementations of DSDV and DSR, derived from the CMU Monarch code. For the MAC and physical layer of our implementation, we used the ns implementation of the $802.11 \mathrm{~b}$ and the built-in radio model that has a radio range of 250 meters.

Our first set of simulations evaluates performance when 50 nodes are subject to different movement models. With our second set of simulations, we measure performance with varying node density ( 25 to 100 nodes) and use the random movement model as the underlying movement pattern of the nodes. For the third set of simulations, we measure how well MRP scales with varying message rates for the random movement model. There are 150 scenario files generated per movement model. We ran both MRP enhanced DSDV and DSDV on each of these files, thereby imposing identical scenarios (node movement, node density, messaging rate) on the two protocols. Section 5 describes and explains our results in detail.

\subsection{Movement Models and Topology}

We investigated the performance of MRP using three different movement models, to understand how sensitive it is to these differences.

1. Random model. This model is the same as in some previous work on ad hoc networks [1,3]. In this model, each node chooses a random destination point within the allowed area to move to. Once the destination is selected,

\footnotetext{
${ }^{1} \mathrm{MRP}$ can produce duplicate packets, which may or may not reach their destination. We register a packet as "received" when the first of these duplicates arrive at their final destination and calculate its latency as the time between when the first packet was sent at the original source and the arrival of this packet at the destination. We ignore all subsequent packets that arrive at the destination, although we keep track of how many such packets arrive to measure the overhead of MRP.
}

the node moves toward that point with a speed distributed uniformly between 0 and some maximum speed. Once the node reaches its destination, it pauses there for a specified pause time. After this time, it chooses a new destination and moves there in memoryless fashion.

2. Soccer player model. This model is somewhat facetiously named after the way children play soccer; here, from the current point, a node picks a random point to move to according to a probability distribution that has a decreasing probability of longer distances being picked. Specifically, the probability density of a node moving a distance $r$ from the current distance falls off as $1 / r$, suitably normalized so that the node does not leave the rectangular field. The model does not bias the direction in which the node moves; all directions are equally likely. Each subsequent move depends only on the current location. We generated movement patterns for this model in the same way as for the random model.

3. Homing pigeon model. This model differs from the previous one in a subtle but important detail. Each node has a randomly chosen "home" location in the field, which never changes during the simulation. Each time a node decides to move (governed by the pause time and speed of movement), it picks a random point that has a probability of being chosen that falls off as $1 / r$, but from the home location. This models a node that ends up always moving around a home region, but also has the property that if it in fact moved far away at some time, will likely come back toward the home location (since the distribution is chosen from the home location) rather than choose another point from the current position.

In all these models, a lower pause time implies a higher degree of mobility. We generated movement patterns for 10 different pause times: 0 (i.e., continuous motion), 30, 60, 120, 300, 600, $800,850,895$, and 900 seconds. The nodes were constrained to move inside a rectangular area of $1500 \times 300 \mathrm{~m}$ for $900 \mathrm{sec}$ onds. With a radio range of 250 meters, this area allowed for paths with a varying number of hops. In addition, during times of mobility, some network partitions occured and some nodes were unreachable for varying durations of time.

We emphasize that these are not necessarily models of any particular real situation, but rather a way by which to obtain an understanding of the factors in any movement model that impact performance. They help us evaluate the sensitivity of the protocol to different movement patterns.

\subsection{Workload}

We chose to use constant bit rate (CBR) traffic sources instead of a feedback-based TCP workload in order to evaluate raw routing protocol performance without having the sources backoff in the face of loss. For the first and second set of simulations (testing performance under different movement mod- 
els and node densities), we generated traffic by selecting 20 random nodes and having them send messages of 3 packets each at a rate of 4 packets per second. Each message transfer started at time uniformly distributed between 0 and 200 seconds. For the third set of simulations (testing scalability), we varied the number of communications between the nodes from 20 to 200 and kept the same message size and the same sending rate for each communication.

\subsection{MRP Parameters}

The choice of an initial value for $h$ (number of permitted relays) depends on how far nodes are expected to move in the system, as a fraction of the total distance between any sourcedestination pair of nodes. In our experiments, we found that even when the probability of a node moving far from its current location is small (but non-zero), an initial value of $h=1$ suffices. That is, there is no need for multiple relaying steps in this situation, although the mechanism may be useful for other movement models where any given node's movement range does not cover the entire field.

We found that the value of $d=1$ is reasonable for DSDV (and better than larger values). DSDV (like other ad hoc protocols) tends to have a significant number of stale routes in its tables during high mobility [1]. By delivering a relay message only if the destination is one hop away, a relay node has a much greater chance to deliver the message before the destination node moves outside of the relay node's "reachable" zone. ${ }^{1}$

\section{Simulation Results}

In this section we present our results for three sets of simulations previously described. The first set evaluates the performance of MRP under different movement models (Sections 5.1 and 5.2). The second set of simulations evaluates MRP performance under different node densities (Section 5.3.1). The third set of simulations evaluates MRP performance under different message rates (Section 5.3.3). In addition we measure the overhead of MRP in Section 5.3.2 to better understand how it scales.

\subsection{Delivery ratio}

\subsubsection{Random Movement Model}

Figure 4 shows the fraction of messages each protocol was able to deliver, as a function of node mobility rate (pause time) when nodes follow a random movement model. The MRPenhanced version of DSDV significantly improves the delivery ratio of messages.

DSDV performs badly when there is high mobility in the network. When nodes continually move, DSDV is able to deliver only fewer than half the packets that are sent. This trend continues until a pause time of 60 seconds, after which point the

\footnotetext{
${ }^{1}$ Our focus was not to determine optimal values for $h$ and $d$, but rather to pick reasonable values and keep them fixed in order to evaluate the effect of other factors such as node movement, node density, and buffer size.
}

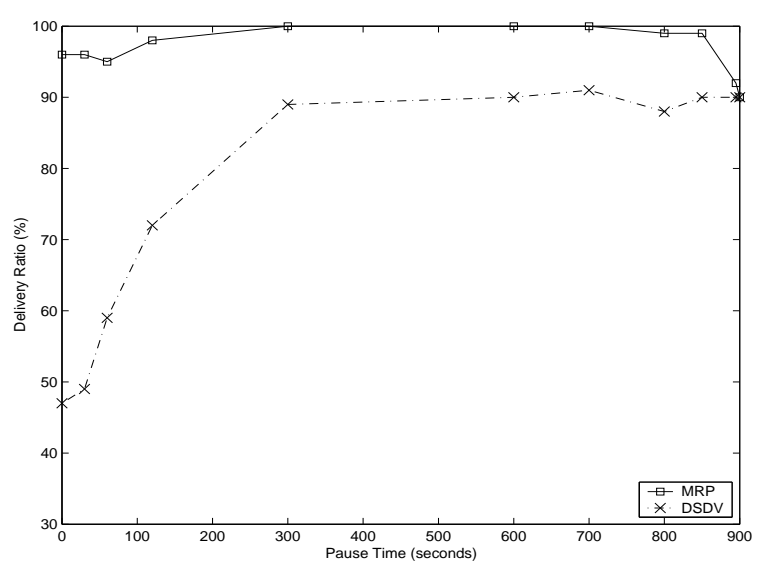

Figure 4: Packet delivery ratio v/s pause time for MRP-enhanced DSDV (MRP) and DSDV on the Random Movement Model for 50 nodes.

performance of DSDV starts improving. The performance of DSDV levels off at around $90 \%$ at a pause time of 300 seconds, before dipping slightly in the completely static case.

For pause times that are between 60 and 300 seconds, we see that although the routing tables are still not able to converge, there is an increasing correlation between the DSDV routing tables and the state of the network. Starting from pause times of about 300 seconds, the routing protocol converges and the delivery ratio is around $90 \%$. After this point, the lost packets are due to two factors. First, some packets simply cannot be delivered. This is due to transient network partitions between a source and destination. Second, routing overhead causes MAC layer collisions and some packets are dropped.

The MRP-enhanced version of DSDV is able to deliver over $94 \%$ of the packets in all cases, except the completely static case. The reason MRP-enhanced DSDV is able to perform so well under a wide range on network conditions is because it delivers packets to the destination when the underlying DSDV protocol is unable to do so.

In the case of complete mobility, MRP delivers around 94\% of the sent packets. This quickly rises to $100 \%$ for less-mobile configurations. In a highly mobile network, each node comes into contact with a large number of nodes by moving to disparate areas of the network. If they encounter the destination, they deliver the message at this point. In a completely static network, the performance of MRP dips to just under $90 \%$, where it matches the performance of DSDV as expected.

\subsubsection{Soccer Player Movement Model}

Figure 5 shows the fraction of messages each protocol was able to deliver when nodes follow the soccer player movement model. Nodes following this movement model have a strong tendency to move in small increments. The MRP-enhanced version of DSDV performs substantially better than DSDV when nodes move.

More interestingly, the performance of MRP on this model is only slightly lower than on the random movement model 


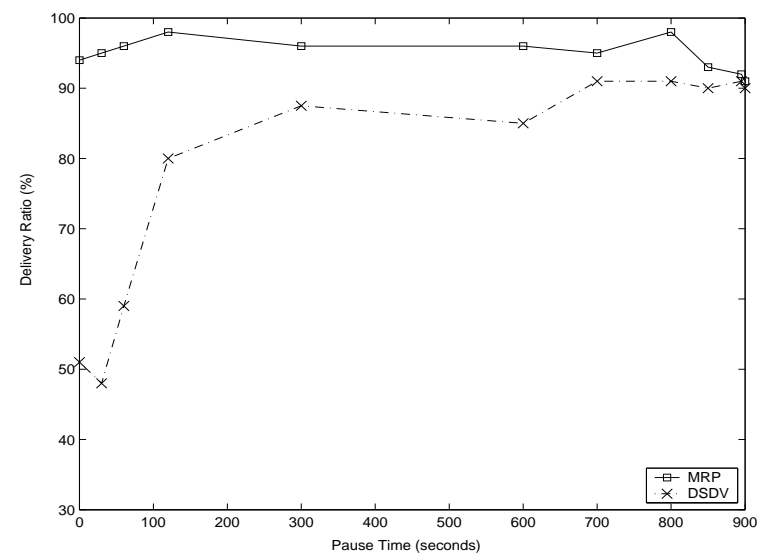

Figure 5: Packet delivery ratio v/s pause time for MRP-enhanced DSDV (MRP) and DSDV on the Soccer Player Model for 50 nodes.

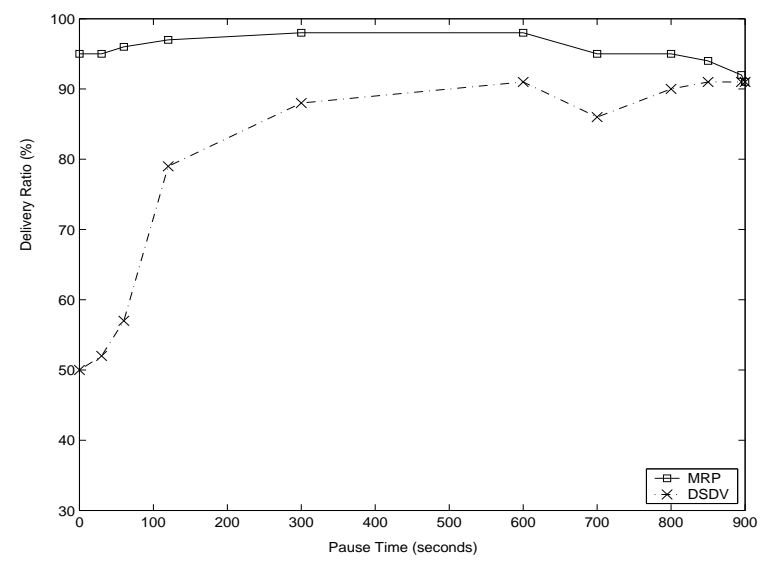

Figure 6: Packet delivery ratio v/s pause time for MRP-enhanced DSDV (MRP) and DSDV on the Homing Pigeon Model for 50 nodes.

when pause times are high. This can be explained by the fact that when the pause times are high, the nodes move only once or twice during a simulation. As nodes move mainly in small increments, there is a high probability that a nodes will move relatively slightly during a simulation. Consequently, the overall topology of the network is similar to that of a static network, in which case the performance of MRP is close to the performance of the underlying DSDV. However, overall it is still a noticeable and significant improvement.

\subsubsection{Homing Pigeon Movement Model}

Figure 6 shows the fraction of messages each protocol was able to deliver when nodes follow the pigeon movement model. In the homing pigeon model, nodes have a strong tendency to move small distances around a random point of origin assigned to each node at the start of each simulation. Again, MRP-enhanced version of DSDV performs substantially better than DSDV except for the completely static case. The performance of MRP on this movement model is slightly lower than on the random movement model, but slightly higher than on the soccer player. Although unintuitive, it appears that nodes in this movement model have longer ranges

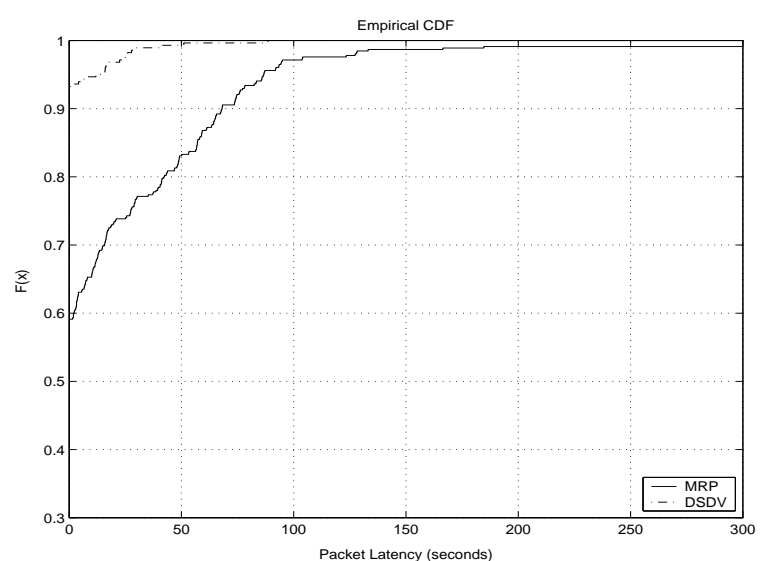

Figure 7: CDF of packet latency for DSDV and MRP-enhanced DSDV for the Random Movement model for a pause time of $60 \mathrm{sec}-$ onds and 50 nodes.

of movements on this model, than on the soccer player model. This is explained by the fact that a nodes next position is calculated from its origin regardless of its current position. Consequently, the total distance traversed by any node is higher than on the soccer player model, and each node is likely to encounter a greater number of nodes as it moves.

The performance of MRP-enhanced DSDV on these movement models shows that MRP is resilient in the face of varied movement models. In each of the models, MRP is able to deliver over $95 \%$ of the packets in most cases. An interesting finding is that even when movement patters are highly localized as in the homing pigeon model, MRP does as well as in the more unconstrained mobility case. An analytic explanation of this effect is an interesting direction for future work.

\subsection{Latency}

Although MRP is able to deliver a substantially higher percentage of packets than vanilla DSDV, this higher delivery ratio comes at the expense of increased latency. This is because the relay nodes carry the packets to their destination by movement rather than passing it along to the next hop in the route by radio transmission. However, we will show that in most cases the packets are delivered within acceptable limits for messaging applications.

\subsubsection{Random Movement Model}

Figure 7 shows the cumulative distribution function (CDF) of packet latencies for DSDV and MRP/DSDV for all the packets in the simulations at a pause time of 60 seconds. The shape of the CDF for 60 seconds presented above is similar to the shape of the distributions for other pause times. The only difference between these curves is that the distributions for the different pause times are centered around different latencies.

As expected, DSDV has a much tighter CDF for packet latency: over $90 \%$ of the packets are delivered within two seconds. It is important to note that this distribution is only based on the number of packets that are delivered. Undeliv- 


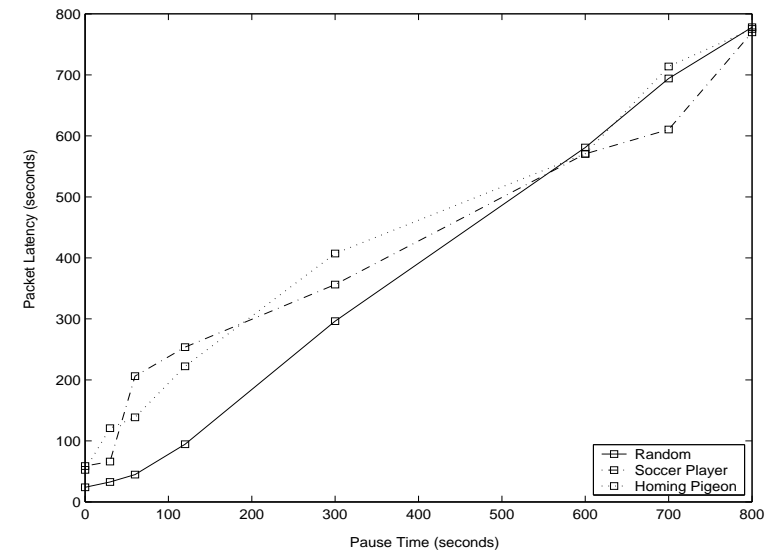

Figure 8: Mean for relay packet delivery latency on the 3 movement models for 50 nodes.

ered packets are not included in this distribution.

For the MRP/DSDV, we see that around $60 \%$ of the packets are delivered within two seconds. These are the packets that were delivered by the underlying DSDV layer. The rest of the packets, mostly relay packets, are spread over a much wider range. By 50 seconds, $90 \%$ of the packets have been delivered, while by 350 seconds, almost all the packets have been delivered. As is evident from this distribution, the MRP is able to deliver packets at a higher delivery ratio, though it trades this capability for increased latency of packet delivery. Still, we see that over $90 \%$ of the packets are delivered in under one minute, and all are delivered in under six minutes. For many messaging applications, where eventual delivery is more important than low latency, these delays are acceptable.

The mean latency for relay packet delivery times over a range of pause times is shown in Figure 8. For the random movement model, the mean delivery time increases linearly with the pause time. This is because at higher pause times the trajectories of the nodes progress slower than at low pause times.

\subsubsection{Soccer Player Movement Model}

The mean latency for packet delivery for the soccer player model is shown in Figure 8. At small pause times, the latencies are comparable to the random movement model. However, at intermediate pause times, the latencies are much higher. At low pause times, nodes move to many destinations during a simulation and consequently can deliver packets quickly. In contrast, at intermediate pause times when nodes move more infrequently, the tendency toward small movements dominates and nodes take longer to encounter their destinations. Hence, the latency sharply rises during these times.

\subsubsection{Homing Pigeon Movement Model}

The mean latency for packet delivery for the homing pigeon model (Figure 8 ) is very similar to the soccer player movement model. However, the delivery times for this model are slightly better. This is because the total per-node movement in this model is longer than in the soccer player model (but small

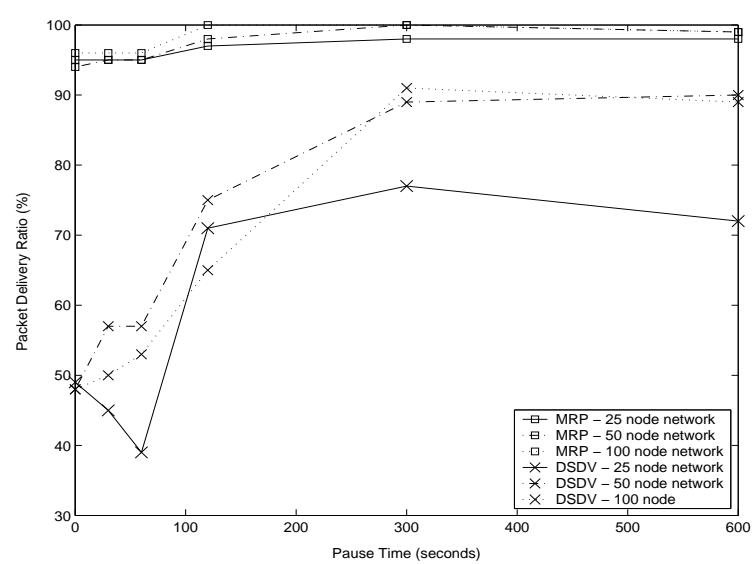

Figure 9: Comparison of delivery ratios vs. pause time for networks of different densities on the Random Movement model.

a ratio compared to the random model). This leads to a similar shaped curve, with slightly better delivery models

An analysis of the mean latencies for packet delivery on the different movement models indicates that the movement patterns do not have a substantial effect on the delivery latency. Movement patterns that have less inherent mobility will have slightly higher delivery latencies for relay packets. However, delivery latencies are all within reasonable bounds for messaging applications.

\subsection{Scalability}

\subsubsection{Node Density}

For an ad hoc protocol to be robust in the face of a variety of scenarios, it is important that it not be adversely affected by an increasing density of nodes in the network. To evaluate the performance of MRP under varying density, we performed the experiments of the previous sections with different number of nodes. We ran simulations on networks of 25 nodes and 100 nodes, representing sparse and dense networks respectively.

Figure 9 shows that the delivery ratio is virtually unaffected by node density. This demonstrates that MRP works to deliver packets even if the number of relay nodes that a packet is handed to is small. This is in stark contrast to the performance of DSDV, also shown in Figure 9, whose performance spans a wide range depending on the density of the nodes. It is significant that MRP is able to adaptively adjust to the shortcomings of DSDV to consistently deliver an extremely high ratio of packets over all pause times and densities.

The delivery times of a relay packet are also affected little by the node density. Figure 10 plots the mean delivery times of relay packets over a range of pause times for 3 different densities over the random movement model. The curves are virtually identical, showing that the time taken to deliver a relay packet does not change significantly with node density.

These results are also significant as they indicate that further fine-tuning of the protocol is possible to reduce overhead. In the current implementation, the node density dictates the num- 


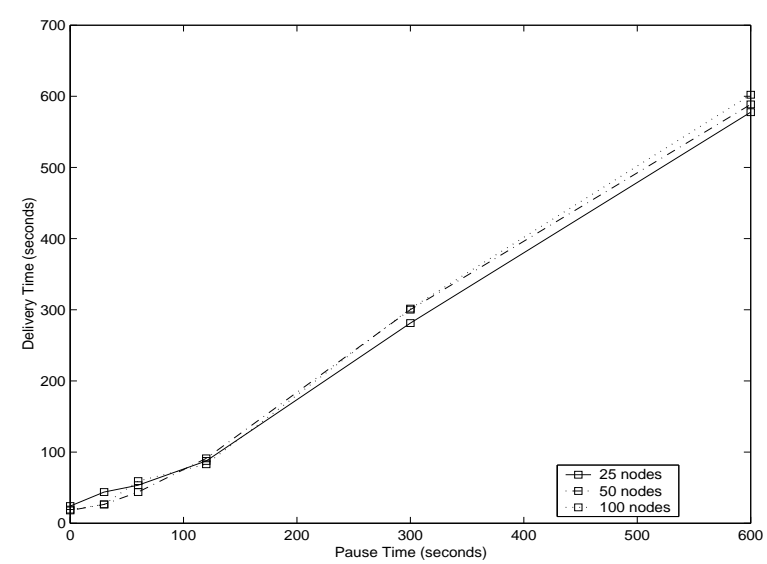

Figure 10: Comparison of mean relay packet delivery latency vs. pause time for networks of different densities on the Random Movement model.

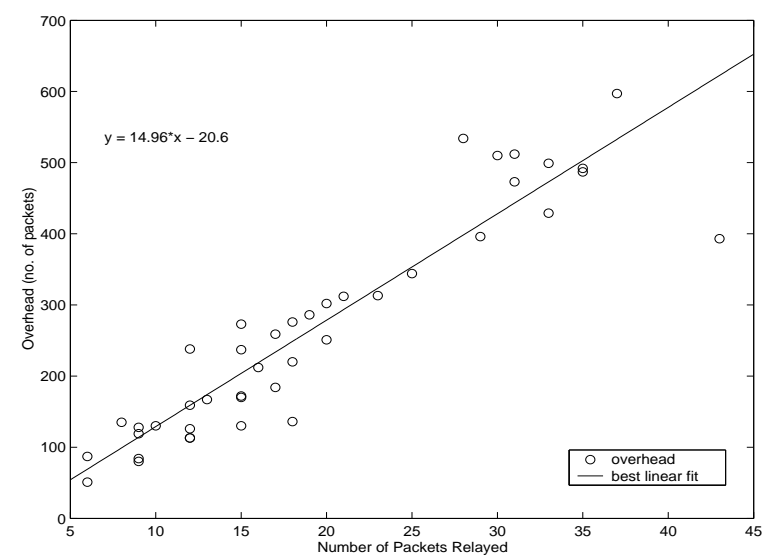

Figure 11: MRP overhead. Each point is the number of broadcasts plus the number of duplicates received over an entire simulation, plotted as a function of the number of relays performed.

ber of nodes that receive a relay packet during a local broadcast. Instead, one could allow nodes to probabilistically accept or reject a packet that is received to relay. Consequently, fewer nodes would relay a packet, lowering the overhead, but keeping the performance characteristics as shown above.

\subsubsection{MRP Overhead}

In this section we examine the overhead for the MRP protocol to determine its impact on the overall bandwidth consumed by routing overhead when MRP is paired with DSDV. Routing overhead in MRP emanates from two sources. The first is at the time of a local broadcast, which is done to distribute relay packets. As this is only one transmission, it can be taken as to be equal to the overhead of a single packet. Overhead is also caused by duplicate packet deliveries at the receiver. Figure 11 shows the MRP overhead for varying numbers of relay packets. This data was extracted from the dataset gathered from running the simulation on the random movement model. As can be seen, the plot can be approximated by a linear fit with a slope of approximately 15 . The variation is

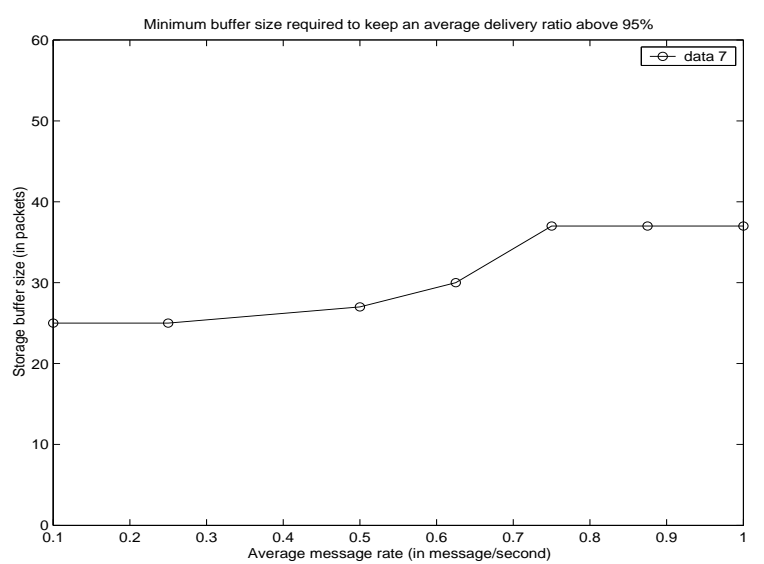

Figure 12: Optimal storage buffer size to keep a $95 \%$ delivery ratio vs. message rates at a pause time of 30 s for 50 nodes on the Random Movement model.

the result of varying numbers of nodes in the vicinity of the node making the local broadcast. While one might be led to believe that linear relationship does not bode well for scalability, when examined within the context of the overhead of the underlying protocol, it is reasonable. In their paper, Maltz et al. [1] document that the routing overhead for DSDV to be 44,000 packets on a similar network for a simulation of the same length. Consequently, when paired with DSDV as the underlying protocol, MRP constitutes less than $1 \%$ of the total routing overhead. As MRP overhead packets consitute such a tiny portion of the total routing overhead, the bandwidth consumed by overhead can be approximated to be only dependent on the underlying protocol. This property of MRP is crucial to its success; if MRP overhead is high, it would saturate the network and reduce the number of packets that are delivered.

\subsubsection{Storage Buffer Size and Message Rate}

To test the performance of MRP under varying message loads, we performed experiments with the random movement model scenarios and with varying message rates. We ran simulations with message rates of $0.1 \mathrm{msg} / \mathrm{s}, 0.5 \mathrm{msg} / \mathrm{s}$ and $1 \mathrm{msg} / \mathrm{s}$. We first experimentally determine an optimal buffer size for different message rates, and then show that with this buffer size, the message rate does not affect the delivery ratio, showing that MRP scales well with increasing message rates.

Buffer Size vs. Message Rate To determine a good buffer size for the nodes, we ran simulations with a pause time of $30 \mathrm{~s}^{1}$ and varied the message rate and the storage buffer size. For each message rate, we determined the minimum buffer size required to keep an average delivery ratio above $95 \%$. Increasing the message rate imposes a higher load on each relay node, showing that the buffer size needs to scale to handle that load, as seen in Figure 12

\footnotetext{
${ }^{1}$ We chose a pause time of 30 s so that the node mobility is high, thereby increasing the number of relay packets in the network.
} 


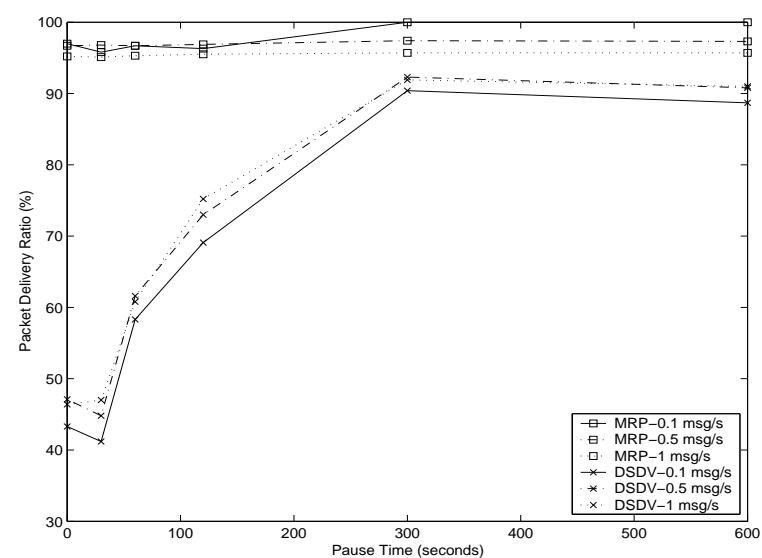

Figure 13: Delivery ratio vs. pause time for different message rates for 50 nodes on the Random Movement model.

A buffer size of 40 packets seems enough to handle all the message rates from 0.1 to $1 \mathrm{msg} / \mathrm{second}$. It is interesting that the required buffer size to keep a high delivery ratio only slowly grows with the message rate.

We find the slow dependence encouraging for MRP scalability in practical situations and we are working on developing an analytic expression in on-going work.

Delivery Ratio vs. Message Rate for a fixed Buffer In this Section, we ran the random movement model scenario files with varying message rates and a fixed buffer size of 40 packets, for both MRP-enhanced DSDV and DSDV. Our aim is to show that for a big enough buffer size, the packet delivery ratio is independent of the message rate. The results are presented in Figure 13. Both DSDV and MRP are unaffected by message rate. This is expected for DSDV since for a given mobility, the factor that affects its delivery ratio is the number of broken routes. No matter what the transmission rate is, if a route is not broken, all the packets will be delivered, and if a route is broken, no packets will be delivered. So the overall ratio should not be affected, as we have shown experimentally. For MRP, a higher message rate means that each node will be storing more packets at a given time. If the right buffer size is found, then no matter what the mobility of the nodes are, the delivery ratio stays the same, which shows that the MRP layer is able to handle varying message loads.

These results suggest that there is an optimal steady-state buffer size.It would be interesting to investigate further how to pick an appropriate buffer size based on the parameters of the system such as active nodes (number of nodes sending a message per second), message size, and node mobility.

\section{Conclusion}

We motivated and presented an opportunistic relay-based approach to message routing in ad hoc networks, integrating routing and storage to improve message delivery ratios. The problem with simply running reliable TCP connections is that it is oblivious to node movement; route failures cause new connections from the messaging applications to be initiated, but these connections will end up breaking over and over as nodes in the network move.

MRP takes advantage of node mobility to disseminate messages to mobile nodes, rather than view mobility as a problem to be solved. Our simulation results under a variety of node movement models demonstrate that this idea can work well for applications that prefer reliability over latency, without adding any significant overhead.

A few opportunities to improve MRP present themselves. We can reduce the number of duplicate packets sent to the final destination by allowing nodes to inconspicuously listen to the network traffic and restrain from delivering a relay packet if it is being delivered by another node. An alternative is for nodes to store and forward packets with a probability that is a decreasing function of the number of neighbors per node.

We found that even when the probability of moving "far" from its current location is "small," and in the homing model, $h=1$ worked well. In general, however, this won't work; if each node moves only a fraction of the total area, then multiple relaying hops will be needed. Determining automated ways of doing this is a topic for future work.

The performance of MRP over other protocols like DSR is also an interesting direction for future work.

\section{References}

[1] Broch, J., Maltz, D. A., Johnson, D., Chu, Y., And JeTCHEVA, J. A performance comparison of multi-hop wireless ad hoc network routing protocols. In Proc. 4th ACM MobiCom (Oct. 1998).

[2] Grossglauser, M., And Tse, D. Mobility Increases the Capacity of Ad-Hoc Wireless Networks. In Proc. IEEE INFOCOM (Apr. 2001).

[3] Johnson, D., And Maltz, D. Dynamic Source Routing in Ad-Hoc Wireless Networks. In In T. Imielinski and H. Korth, editors, Mobile Computing (1996), Kluwer Acadmic Publishers.

[4] Kotz, D., Gray, R., Nag, S., Rus, D., Chawla, S., And CyBeno, G. Agent Tcl:Targetting the Needs of Mobile Computers. IEEE Internet Computing (July/August 1997).

[5] LI, Q., AND RUS, D. Sending Messages to Mobile Users in Disconnected Ad-Hoc Wireless Networks. In Proc. 6th ACM MobiCom (Aug. 2000).

[6] ns-2 (network simulator and nam network interface). http: //www.isi.edu/nsnam/ns/.

[7] Park, V. D., And Corson, M. S. A Highly Adaptive Distributed Routing Algorithm for Mobile Wireless Networks. In Proc. IEEE INFOCOM (Apr. 1997).

[8] Perkins, C., And Bhagwat, P. Highly Dynamic DestinationSequenced Distance-Vector Routing (DSDV) for Mobile Computers. In Proc. ACM SIGCOMM (Oct. 1994), pp. 234-244.

[9] Perkins, C. E., AND Royer, E. M. Ad Hoc On Demand Distance Vector (AODV) Algorithm. In Proc. 2nd IEEE Workshop on Mobile Computing Systems and Applications (WMCSA) (Feb. 1999). 